\title{
Efectos de la intensificación ganadera sobre la eficiencia en el uso de la energía fósil y la conservación del pastizal en la cuenca baja del río Salado, provincia de Buenos Aires, Argentina
}

Jacobo, E.; A. Rodríguez, J. González y R. Golluscio.

\begin{abstract}
RESUMEN
Con el objetivo de explorar las consecuencias sobre la sustentabilidad ambiental del proceso de intensificación de la ganadería bovina de la cuenca del Salado, se comparó la proporción y condición del pastizal natural, la cantidad de energía fósil consumida por unidad de superficie y de producto y la eficiencia en el uso de energía fósil de13 establecimientos con distinta intensidad en el uso de insumos para la alimentación del ganado. La energía fósil consumida por unidad de superficie mostró un rango entre 600 y $3600 \mathrm{MJ}$ hectárea-1 año $^{-1}$, la consumida por unidad de producto se ubicó entre 0,01 y $8,49 \mathrm{MJ} \mathrm{kg}^{-1}$ de peso vivo de carne bovina y la eficiencia en el uso de energía resultó entre 0,92 y $1973 \mathrm{MJ}$ producidos por MJ consumidos. El consumo de energía fue inferior y la eficiencia en su uso fue superior en los establecimientos que conservan una elevada proporción de pastizal en buena condición, como consecuencia del adecuado manejo del pastoreo respecto de la de aquellos que usaron mayor cantidad de insumos para la alimentación. El actual proceso de intensificación está disminuyendo la eficiencia energética de los sistemas de producción de carne, lo que sugiere una alerta para la sustentabilidad ambiental.
\end{abstract}

Palabras clave: Pampa Inundable, escala establecimiento, indicadores de sustentabilidad, agroecología

Jacobo, E.; A. Rodríguez, J. González and R. Golluscio, 2016. Effects of livestock intensification on fossil energy use efficiency and rangeland conservation in the lower basin of the Salado river, Buenos Aires province, Argentine. Agriscientia 33 (1): 1-14

\section{SUMMARY}

The aim of the study was to explore the impact of the current process of intensification in cattle production systems on the environmental sustainability 
in the Salado basin region. We compared the proportion and condition of grasslands, fossil energy consumption per unit of area and per unit of product and fossil energy use efficiency on13 livestock farms that used different input levels for cattle feeding. Fossil energy consumption per unit of area ranged from 600 to 3600 megajoules (MJ) ha ${ }^{-1} \mathrm{yr}^{-1}$, fossil energy consumption per unit of product from 0.01 to $8.49 \mathrm{MJ} . \mathrm{kg}^{-1}$ of live weight, and fossil energy use efficiency from 0.92 to $1973 \mathrm{MJ}$ of energy produced per MJ of energy consumed. Fossil energy consumption was lower and fossil energy use efficiency was higher on farms with a large proportion of rangeland in good condition as a consequence of appropriate grazing management with respect to higher feed-input farms. The current process of intensification is causing a decrease in the energy efficiency of meat production systems, which might affect environmental sustainability

Keywords: Flooding Pampas, farm scale, sustainability indicators, agroecology

Jacobo, E.; A. Rodríguez, J. González y R. Golluscio: Universidad de Buenos Aires, Facultad de Agronomía, Departamento de Producción Animal. Ciudad Autónoma de Buenos Aires, Argentina. Correspondencia a: ejacobo@agro.uba.ar

\section{INTRODUCCIÓN}

El proceso de expansión agrícola ocurrido en la Argentina en las últimas dos décadas generó un drástico cambio en el uso del suelo en las áreas de producción agropecuaria extensiva, con características particulares según la zona considerada. En la pradera pampeana, donde la agricultura se incorporó a principio del siglo XX a través de rotaciones entre la agricultura y la ganadería, se reemplazó la superficie sembrada con pasturas por cultivos de cosecha; por su parte, en áreas boscosas o con suelos limitantes para la actividad agrícola, los cultivos anuales están reemplazando a la vegetación natural (Paruelo et al., 2005). La expulsión de la ganadería de las zonas con mayor aptitud agrícola disminuyó la superficie destinada a esta actividad en el país (Rearte, 2010) y aumentó la presión de pastoreo sobre la vegetación natural de áreas con limitantes para la siembra de cultivos.

La intensificación de la producción agropecuaria se sustenta en la especialización productiva, en el aumento de la productividad del trabajo a través de la simplificación del manejo de los cultivos o del ganado y en una gran utilización de insumos externos, como fertilizantes, agroquímicos o alimentos concentrados. Este proceso puede resultar nocivo para el ambiente y es una de las principales causas de la pérdida de los servicios ecosistémicos de regulación y soporte (Power, 2010). Por ello, uno de los desafíos actuales en términos de manejo y estudio de los ecosistemas es valorar cuanti- tativamente la provisión de servicios ecosistémicos mediante distintos procedimientos, siendo uno de ellos la cuantificación de los flujos de energía en los agro-ecosistema (Ferraro, 2011).

La pampa inundable, cuyas características ambientales limitan severamente el uso agrícola, es un área de 9 millones de hectáreas ubicada al este de la provincia de Buenos Aires, cuya mayor superficie (6,5 millones de ha) está constituida por la cuenca baja del río Salado. Esta región, que aún conserva entre $53 \%$ y $89 \%$ de pastizales naturales o seminaturales, es una de las principales zonas ganaderas del país, ya que aloja más del 20\% del stock bovino nacional (Vázquez \& Rojas, 2006). La carga animal en el año 2003 era de 1,1 EV ha-1 (Vázquez \& Rojas, 2006), y se incrementó a 1,54 EV ha $^{-1}$ en el año 2007 (Vázquez \& Rojas, 2008). Las consecuencias del proceso de expansión agrícola e intensificación de la producción agropecuaria en esta región son: i) el deterioro del suelo y una baja estabilidad y productividad de los recursos forrajeros al reemplazar las comunidades naturales en ambientes con limitaciones (Jacobo \& Rodríguez, 2009); ii) la drástica reducción de la riqueza, la extinción local de las forrajeras nativas más valiosas, la disminución de la productividad primaria y la alteración de su estacionalidad debido a la pulverización con glifosato sobre las comunidades no inundables (Rodríguez \& Jacobo, 2010; 2013); iii) el deterioro de la vegetación natural por aumentos de carga por encima de su receptividad (Jacobo \& Rodríguez, 2009); iv) la reducción de la produc- 
tividad primaria neta como consecuencia del proceso de degradación (Recavarren \& Martinefsky 2009); v) el aumento en el uso de insumos para la alimentación del ganado (Vázquez et al., 2008a). Todos estos cambios alertan sobre la necesidad de detener la pérdida de biodiversidad y el deterioro del pastizal natural, principal recurso forrajero de la ganadería pastoril de la cuenca baja del río Salado.

Los ecosistemas de pastizales naturales ofrecen variados bienes y servicios que brindan beneficios a la sociedad, tanto de manera directa mediante la provisión de alimentos, como de manera indirecta mediante el control del clima, la mitigación de inundaciones y sequías, y el ciclado de materia orgánica, entre otros (Fisher et al., 2009). La biodiversidad de los pastizales naturales es considerada un servicio ecosistémico de soporte de las funciones de producción (Altesor, 2010), a través de su relación con diversos procesos tales como la fijación de carbono (Soussana et al., 2010), el reciclado de nutrientes y la estabilidad ecosistémica (Wrage et al., 2011), la resistencia a la invasión de especies exóticas (Perelman et al., 2007) y el control de la erosión (Balvanera et al., 2006). Para garantizar la provisión de estos bienes y servicios de los pastizales naturales, es imprescindible la conservación de la salud de los ecosistemas (Briske et al., 2005), ya que los pastizales saludables son considerablemente más productivos, estables y resilientes que los pastizales degradados (Milchunas \& Lauenroth, 1993). Dado que los cambios en la condición del pastizal ocurren lentamente, a menudo el deterioro no se detecta hasta que está muy avanzado (Savory \& Butterfield, 1999); por lo tanto, al intentar maximizar los resultados a corto plazo se puede degradar el pastizal y comprometer la productividad futura (Teague et al., 2011).

Simultáneamente con el deterioro del pastizal natural (Recavarren \& Martinefsky, 2009) y la reducción de la superficie ganadera (Rearte, 2010), ocurrió un aumento de la utilización de insumos y en consecuencia del consumo de combustibles fósiles (Frank \& Viglizzo, 2010). El mayor uso de insumos ha permitido sostener o incluso aumentar la productividad ganadera. Sin embargo, esto podría aumentar la cantidad de energía fósil requerida para producir una unidad de producto y reducir, en consecuencia, la eficiencia energética, considerada un indicador de sustentabilidad de los agroecosistemas (Halberg et al., 2005). En la producción ganadera, el mayor consumo energético proviene de la alimentación del ganado, incluyendo el gasto energético para su producción y transporte. Los trabajos donde se cuantifica la cantidad de energía fósil necesaria para producir un kilo de peso vivo bovino muestran que ésta es altamente variable y que las características del forraje utilizado en la alimentación del ganado vacuno son determinantes de la eficiencia energética de los sistemas (Cederberg \& Darelius, 2002; Cedelberg \& Stadig, 2003; Casey \& Holden, 2006; Williams et al., 2006; Ogino et al., 2007; Pelettier et al., 2010) ya que cuanto mayor sea la proporción de forraje que requiera pocos insumos para su producción y transporte, más eficiente energéticamente y, por lo tanto, más sustentable resultará la producción ganadera. Por ejemplo, en dos áreas de la Argentina cuyos sistemas ganaderos se basan en el pastizal natural (cuenca del Salado y San Luis), la eficiencia energética calculada como indicador de sustentabilidad ambiental fue considerada alta (Cieza \& Flores, 2007; Gil et al., 2009).

El objetivo de este trabajo fue explorar las consecuencias sobre la sustentabilidad ambiental del proceso de intensificación de los sistemas de producción de carne bovina de la cuenca del Salado. Para ello se comparó la proporción y condición del pastizal natural, la cantidad de energía fósil consumida por unidad de superficie y por unidad de producto y la eficiencia en el uso de la energía fósil de 13 establecimientos ganaderos que utilizaron distinto nivel de uso de insumos para la alimentación de los animales.

\section{MATERIALES Y MÉTODOS}

\section{Área de estudio}

El estudio se realizó en la cuenca baja del río Salado, subregión de aproximadamente 6,5 millones de hectáreas, ubicada al sudeste de la región pampeana. El clima es templado húmedo, con un promedio anual de precipitaciones que varía de $1000 \mathrm{~mm}$ en el norte a $850 \mathrm{~mm}$ en el sur y con un rango de temperaturas medias mensuales de 6,8 ${ }^{\circ} \mathrm{C}$ en julio-agosto a $21,8{ }^{\circ} \mathrm{C}$ en enero. El relieve de la subregión es casi plano -las pendientes no superan el $3 \%-y$ presenta una predominancia de suelos salinos y/o alcalinos con drenaje deficiente, lo que determina la ocurrencia de anegamientos frecuentes (Vázquez et al., 2008a). La productividad primaria neta aérea de los pastizales de la región es de alrededor de $2000 \mathrm{~kg} \mathrm{MS} \mathrm{ha-1}$ año-1 en comunidades halomórficas (estepas de halófitas), de $6000 \mathrm{~kg} \mathrm{MS} \mathrm{ha}^{-1} \mathrm{año}^{-1}$ en comunidades hidromórficas (praderas de higrófitas) y de alrededor de $5000 \mathrm{~kg} \mathrm{MS} \mathrm{ha-1}^{-1} \mathrm{año}^{-1}$ en comunidades no halomórficas ni hidromórficas (pradera húmeda de mesófitas) (Hidalgo \& Cahuepé, 1991). La actividad tradicional ha sido la cría de ganado bovino, aunque 
últimamente también se realiza recría e invernada.

Esta subregión ha sido dividida en subzonas según la proporción de superficie de pastizal que conservan y el tipo de actividades productivas predominantes (Vázquez \& Rojas, 2006). Las dos subzonas más extensas, que conservan la mayor superficie de pastizal natural, entre 89 y $86 \%$, y presentan una relación similar entre la cantidad de vacas y la cantidad total de animales de 0,48 y 0,50, según el censo 2002, son la "ganadera de la costa", que incluye a los partidos lindantes con la bahía de Samborombón, y la "de derrames", que incluye a los partidos ubicados en el centro de la cuenca del Salado (Vázquez \& Rojas, 2006). Estas dos subzonas son homogéneas entre sí a escala de paisaje, con dominancia de suelos hidro-halomórficos y con una proporción de suelos no inundables y profundos que no supera el 33\% (Vázquez et al., 2008b).

Se seleccionaron 13 establecimientos productivos (Figura 1) de estas dos subzonas, procurando incorporar establecimientos de diferente tamaño, tipo de actividad ganadera y estrategia alimenta- ria (según las proporciones relativas de suelos no inundables, de pastizal natural y de recursos forrajeros implantados, y el aporte de suplementos utilizados en la alimentación). Si bien en la cuenca del Salado hay establecimientos especializados en el engorde a corral y por lo tanto constituyen el límite superior del proceso de intensificación, se han excluido de este estudio para focalizarlo en la producción de carne de base pastoril. Los 13 establecimientos ganaderos seleccionados son representativos del universo de la cuenca del Salado, de acuerdo a su tamaño, la proporción de suelos no inundables, la proporción de la superficie en que se conserva el pastizal natural, la metodología de manejo del pastizal natural (pastoreo controlado o no controlado, según Deregibus et al. 1995), la cantidad de suplementos ofrecidos, la terminación a pasto o a corral, la producción de carne y el tipo de producción (Tabla 1). Si bien el estudio se realizó en dos subzonas homogéneas entre sí a escala de paisaje, la proporción de suelos no inundables fue muy distinta a escala de los establecimientos, y por ende su potencial productivo, ya que en los suelos no inundables la siembra de pasturas y ver-

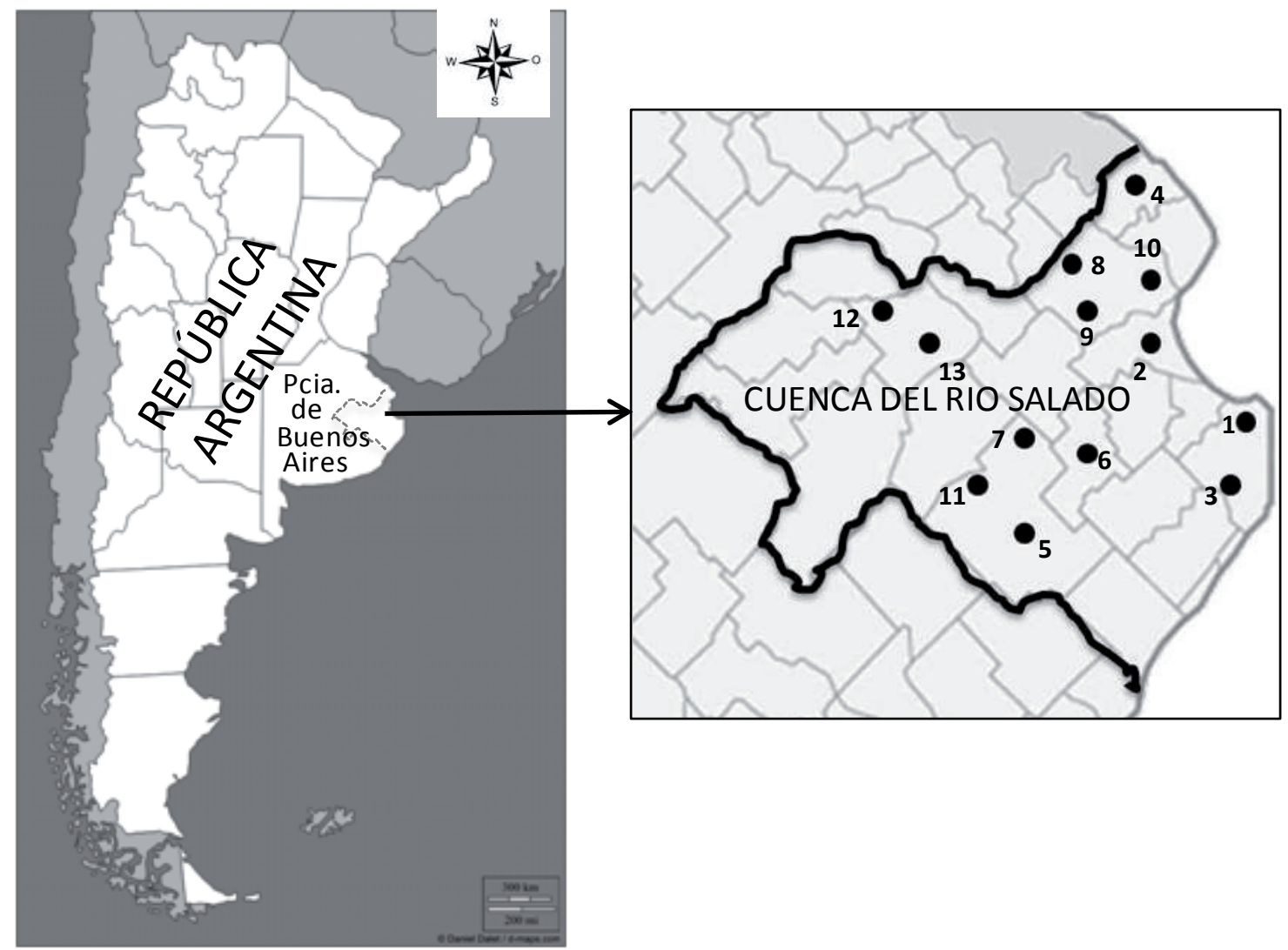

Figura 1: Ubicación de la cuenca del Salado en la República Argentina. A la derecha se indican los 13 establecimientos que constituyeron los casos de estudio con un círculo negro y el número que los identifica. 


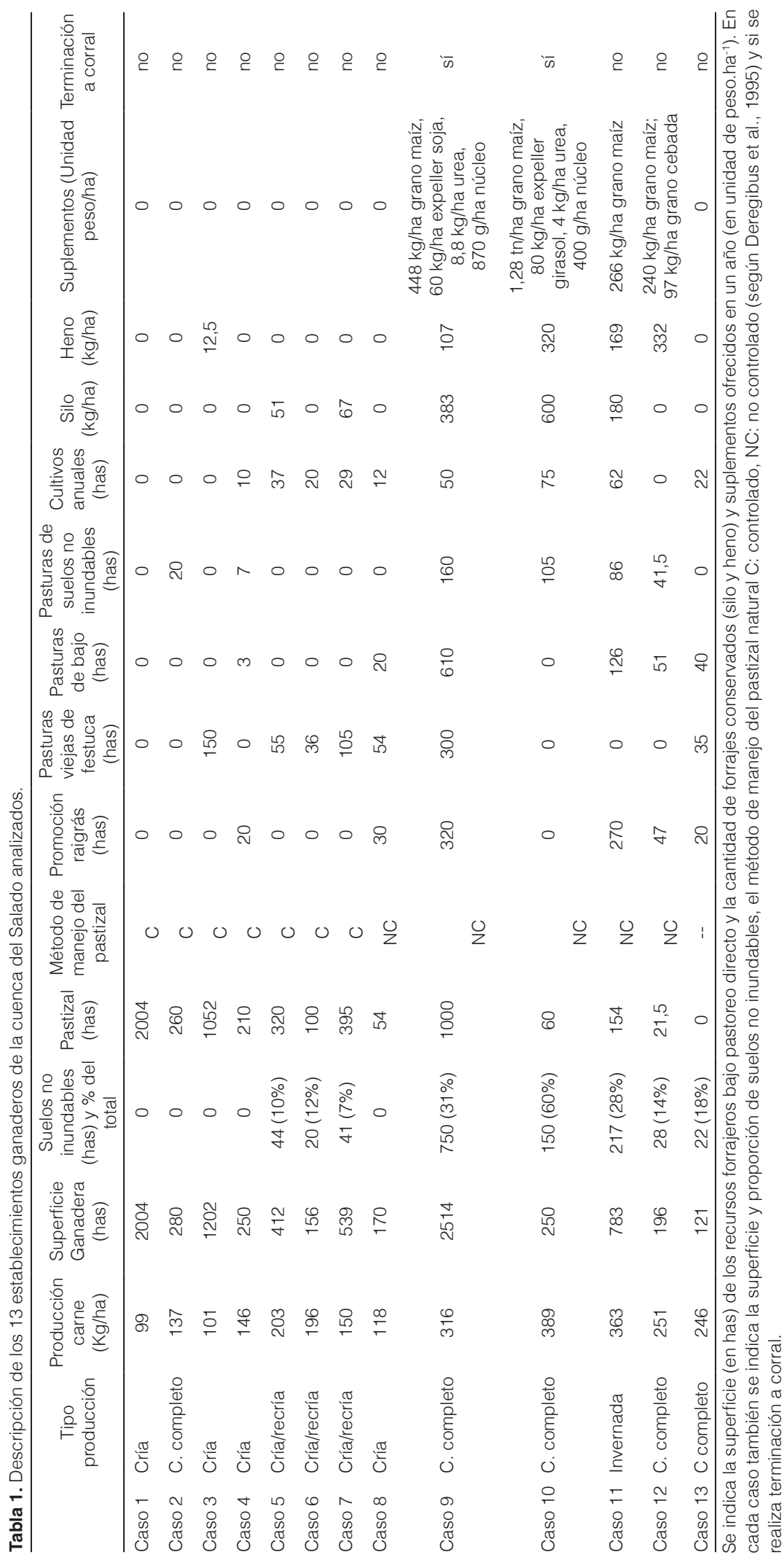


deos es una práctica recomendable. En efecto, los establecimientos 8,9 y 11, donde la producción de carne superó los $300 \mathrm{~kg} \mathrm{ha}^{-1}$, tienen 31, 60 y 28\% de sus suelos sin problemas de hidromorfismo, respectivamente, mientras que en el resto de los establecimientos la proporción de suelos no inundables no superó el 18\%. El caso 9 constituye una excepción para la zona, ya que la proporción de suelos no inundables es muy superior a la característica en el área, por estar ubicado en el albardón de una gran laguna. Por su parte, el caso 11 es el único donde la actividad ganadera es la invernada o engorde de terneros/as, cuya eficiencia en la conversión de pasto a producto animal es superior a aquellas actividades que incluyen la etapa de gestación y cría de terneros (cría, cría y recría y ciclo completo). Adicionalmente, en los casos 8 y 9 , la "terminación" o final del período de engorde de los animales, se realiza mediante el confinamiento en corrales donde se ofrecen granos y otros suplementos, una parte de los cuales se producen fuera de los establecimientos.

\section{Fuentes de información}

Se realizaron encuestas semiestructuradas para recolectar la información correspondiente al ejercicio comprendido entre julio de 2013 y junio de 2014, con el objetivo de caracterizar a cada establecimiento según el uso del suelo, la actividad ganadera, las labores e insumos utilizados en los recursos forrajeros, la alimentación suplementaria y la producción de carne.

Respecto del uso del suelo, se estimó la superficie asignada a cada recurso forrajero: pasturas viejas de Festuca arundinacea Schreb. (festuca), pasturas de suelos bajos [Agropiron elongatum (Host) P.B. (agropiro) y Lotus corniculatus L. (trébol de olor amarillo)], pasturas de suelos no inundables [en las posiciones más altas: Medicago sativa L. (alfalfa) o Trifolium pratense L. (trébol rojo), Trifolium repens L. (trébol blanco), Dactylis glomerata L. (pasto ovillo) o Bromus unioloides H.B.K. (cebadilla); en las posiciones intermedias: festuca, trébol blanco y/o trébol de olor amarillo)], promoción de Lolium multiflorum Lam (raigrás anual), cultivos forrajeros anuales [Avena sativa L. (avena), Hordeum vulgare L. (cebada), Zea maize L. (maíz), Sorghum bicolor (L.) Moench (sorgo)], pastizal natural sobre suelos inundables y pastizal natural sobre suelos no inundables.

Durante la primavera de los años 2014 y 2015 se realizaron censos de cobertura vegetal y proporción de especies en los potreros con pastizal natural o seminatural de cada uno de los estable- cimientos. Estos relevamientos se realizaron ubicando al azar una transecta de 7,5 $\mathrm{m}$ de longitud en cada uno de los potreros. A lo largo de cada transecta se registró la ocurrencia de cada especie cada $5 \mathrm{~cm}$ a nivel del suelo. Con esos datos se obtuvo la cobertura basal total y la de especies nativas o naturalizadas con valor forrajero. Se consideró de valor forrajero a las especies con un valor de tres o más, según la categorización realizada por Cahuepé et al. (1985). Siguiendo la metodología propuesta por Viglizzo (2013), no se consideraron como pastizal a aquellos lotes que presentaron más del $50 \%$ de especies exóticas, y se corrigió la cobertura de especies de valor forrajero por la proporción de exóticas cuando su proporción fue inferior al 50\%, según lo siguiente: si la proporción de especies exóticas estaba entre 11 y $20 \%$, el factor de corrección a restar de la cobertura fue 2,5\%; si estaba entre 21 y $30 \%$ se restó $5 \%$; entre 31 y $40 \%$ se restó $7,5 \%$, y entre 41 y $50 \%$ se restó $10 \%$.

\section{Cálculos de las variables utilizadas}

Condición y participación del pastizal natural en la alimentación animal

La condición de pastizal se calculó como el producto de la cobertura basal total y la de especies de valor forrajero corregido. Para cada establecimiento se obtuvo el valor de condición del pastizal como el promedio ponderado por la superficie de todos los potreros evaluados. Para obtener un indicador que dé cuenta de la importancia del pastizal natural en la alimentación animal, se multiplicó la proporción de pastizal natural de cada establecimiento por la condición del pastizal natural (Tabla 2).

\section{Consumo de energía fósil para alimentación animal}

La definición de los límites del sistema es uno de los aspectos más importantes para la realización de los cálculos de un análisis energético. Con respecto a las entradas de energía (inputs), se incluyó el uso directo de energía en cada campo (gasoil), la energía utilizada para la fabricación de los insumos utilizados en el sistema (costo energético de producción de semillas, gasoil, fertilizantes y plaguicidas) y la energía utilizada para la elaboración de la maquinaria utilizada. No se consideraron los gastos energéticos de almacenamiento (silo bolsa) y de trasporte de insumos y productos, la energía asociada al trabajo humano, ya que en sistemas extensivos representan menos del $0,2 \%$ del total de entrada de energía (Zentner et al., 2004), ni los gastos en productos zoosanitarios dado que los coeficientes energéticos no se encontraron dispo- 
Tabla 2. Proporción y condición del pastizal natural de los 13 establecimientos ganaderos de la cuenca del Salado analizados

\begin{tabular}{cccc}
\hline & $\begin{array}{c}\text { Proporción } \\
\text { Pastizal (\%) }\end{array}$ & $\begin{array}{c}\text { Condición } \\
\text { pastizal (\%) }\end{array}$ & Proporción ${ }^{*}$ Condición \\
\hline Caso 1 & 100 & 75 & 75 \\
Caso2 & 93 & 70 & 65 \\
Caso 3 & 88 & 62 & 54 \\
Caso 4 & 84 & 76 & 64 \\
Caso 5 & 78 & 77 & 60 \\
Caso 6 & 74 & 71 & 52 \\
Caso 7 & 73 & 72 & 53 \\
Caso 8 & 16 & 39 & 6 \\
Caso 9 & 43 & 37 & 16 \\
Caso 10 & 24 & 39 & 9 \\
Caso 11 & 13 & 37 & 5 \\
Caso 12 & 11 & 34 & 4 \\
Caso 13 & 0 & 0 & 0 \\
\hline
\end{tabular}

Condición del pastizal (cobertura basal total ${ }^{*}$ cobertura de especies de valor forrajero * 100) proporción * condición (proporcióncondición/100).

nibles. Tampoco se tuvieron en cuenta los gastos energéticos asociados a la estructura de los establecimientos, tales como la calefacción y la energía eléctrica utilizada para el hogar y los gastos de combustible debido a traslados. Con respecto a las salidas de energía (outputs) se consideró la contenida en la carne producida (incluye ventas y diferencias de inventario).

Con respecto a los coeficientes energéticos utilizados para estimar los inputs de energía, existe gran variedad de opciones para utilizar sin que exista un protocolo metodológico que permita discernir entre uno y otro (Perez Neira, 2010). En este trabajo se utilizaron los coeficientes citados por lermanó (2015). En el caso de las pasturas se amortizó la energía total utilizada en su período de vida útil. La energía fósil total utilizada en cada establecimiento productivo se expresó en megajulios de energía por hectárea ganadera $\left(\mathrm{MJ} \mathrm{ha}^{-1}\right)$ y se separó de acuerdo a la utilizada con distintos objetivos: i) implantación de pasturas y verdeos; ii) regulación biótica (control de malezas, plagas y enfermedades); iii) mantenimiento o aumento de la fertilidad del suelo; y iv) suplementación animal con forrajes conservados, granos y otros.

Con el objeto de comparar el consumo de energía fósil con otros sistemas de producción de carne, ésta se expresó como la cantidad total de energía fósil utilizada para producir un kilogramo de carne (MJ kg-1 de peso vivo).

\section{Eficiencia en el uso de la energía fósil}

La eficiencia en el uso de la energía se calculó como el cociente entre la energía contenida en el producto y la energía consumida en un lapso determinado (Pimentel et al., 1973). Los resultados obtenidos se compararon con los inversos multiplicativos de los valores de referencia elaborados por Viglizzo et al. (2006) para diagnosticar sustentabilidad ambiental en la región pampeana, ya que estos autores calcularon la eficiencia en el uso de la energía como el cociente entre la energía fósil consumida en un año y la energía contenida en el producto producido en el mismo período.

\section{Análisis de la información}

Se realizó un ordenamiento mediante análisis de componentes principales (PCA) (McCune \& Mefford, 1999) de los establecimientos ganaderos de acuerdo a las variables relevadas y/o calculadas: proporción de pastizal natural; condición del pastizal natural; energía fósil utilizada con distintos objetivos (implantación de forrajeras; regulación biótica o sea control de malezas, plagas y enfermedades; mantenimiento de la fertilidad y suplementación); producción de carne por hectárea y energía utilizada por unidad de producto (MJ kg-1 de peso vivo). Para ello se relativizaron las variables mediante el ajuste al desvío estándar. Posteriormente se realizó un análisis de conglomerados (McCune \& Mefford, 1999) con el objetivo de agrupar los establecimientos productivos en grupos similares (con un porcentaje de similitud mayor al 63\% de la variación total). Finalmente se puso a prueba la diferencia entre los grupos resultantes mediante MRPP (Multiresponse permutation procedure) (Biondini et al., 1988).

Se realizó la regresión entre la proporción de pastizal natural y su condición para cada uno de los establecimientos ganaderos analizados.

\section{RESULTADOS Y DISCUSIÓN}

El ordenamiento de los casos de estudio de acuerdo a las variables analizadas (consumo de energía fósil discriminada según objetivos de producción; proporción de pastizal natural; condición del pastizal natural; producción de carne por hectárea; consumo de energía fósil por unidad de producto) permitió explicar el 83\% de la variabilidad total mediante los dos primeros ejes (Figura 2). El primer eje, que incluye $67 \%$ de la variación total de los datos, representa un gradiente de intensificación y ubica a la izquierda (corresponde a los valores negativos del eje) a los establecimientos con mayor proporción de uso de pastizal natural, y a la derecha (corresponde a los valores positivos) a los establecimientos con mayor consumo de ener- 


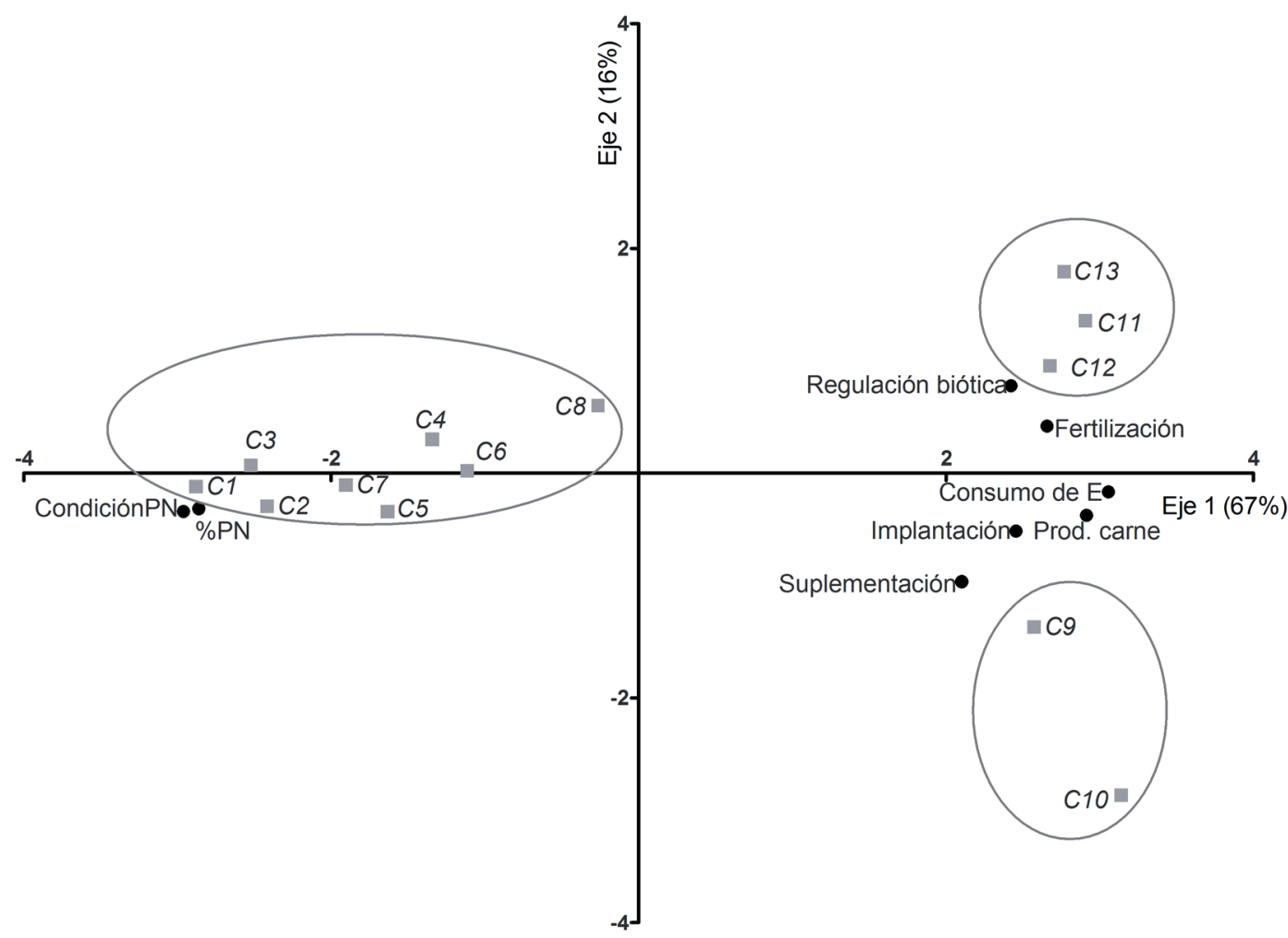

Figura 2. Ordenamiento (análisis de componentes principales) y grupos establecidos (análisis de conglomerados) de los 13 establecimientos ganaderos de la cuenca del Salado (C1 a C13). Se insertan las variables: proporción de pastizal natural (\%PN); condición del pastizal natural (Condición PN); la cantidad de energía fósil consumida, en MJ.ha-1.año-1, para implantación, regulación biótica, fertilización y suplementación; energía fósil consumida en $\mathrm{MJ} \mathrm{kg}^{-1}$ de carne producida (Consumo de E) y producción de carne en kg.ha-1. año-1 (Prod. carne).

gía fósil. El segundo eje (16\% de la variabilidad) discrimina entre los establecimientos más intensificados, ubicando hacia los valores positivos a los establecimientos que utilizan la energía fósil, principalmente en fertilizantes y agroquímicos, y hacia los valores negativos a los que la utilizan principalmente en suplementación. El análisis de conglomerados coincidió con la discriminación surgida del ordenamiento, ya que con un porcentaje de encadenamiento mayor al 62,5\%, separó tres grupos: un primer grupo que integra la mayor cantidad de establecimientos (ocho casos) corresponde a los casos que realizan un menor consumo de energía fósil, un segundo grupo (tres casos) que corresponde a los establecimientos que utilizan la energía fósil en sembrar pasturas y verdeos, y un tercer grupo (dos casos) que, además de sembrar pasturas y verdeos, ofrece una importante cantidad de suplementos a través de la alimentación a corral. El análisis MRPP confirmó este agrupamiento ( $\mathrm{T}=$ $-4,19 ; p<0,001)$.

La cantidad total de energía fósil utilizada en la alimentación del ganado en los establecimientos analizados, expresada en MJ ha-1 ganadera, se ordenó según un gradiente de producción secundaria o salida energética (Figura 3). La energía fósil consumida en los establecimientos que integran el grupo de menor consumo (casos 1 al 8) no superó los $600 \mathrm{MJ}_{\text {ha-1 }}$ año-1, mientras que la consumida en los establecimientos de los dos grupos más intensificados (casos 9 al 13) se ubicó entre 1600 y $3600 \mathrm{MJ} \mathrm{ha}^{-1}$ año-1. El tipo de sistema de producción ganadera de los establecimientos del grupo de menor consumo es la cría o la críarecría, mientras que el de los establecimientos de mayor consumo es el ciclo completo o la invernada, con la única excepción del caso 2, que realiza ciclo completo aunque se ubica en el grupo de menor consumo (Tabla 1). Si bien la energía consumida en estos últimos superó varias veces a la utilizada en el grupo de menor consumo, todos los establecimientos registraron un consumo de energía menor a $5000 \mathrm{MJ} \mathrm{ha}^{-1}$ año-1, que es el rango de menor consumo energético estimado por Viglizzo 


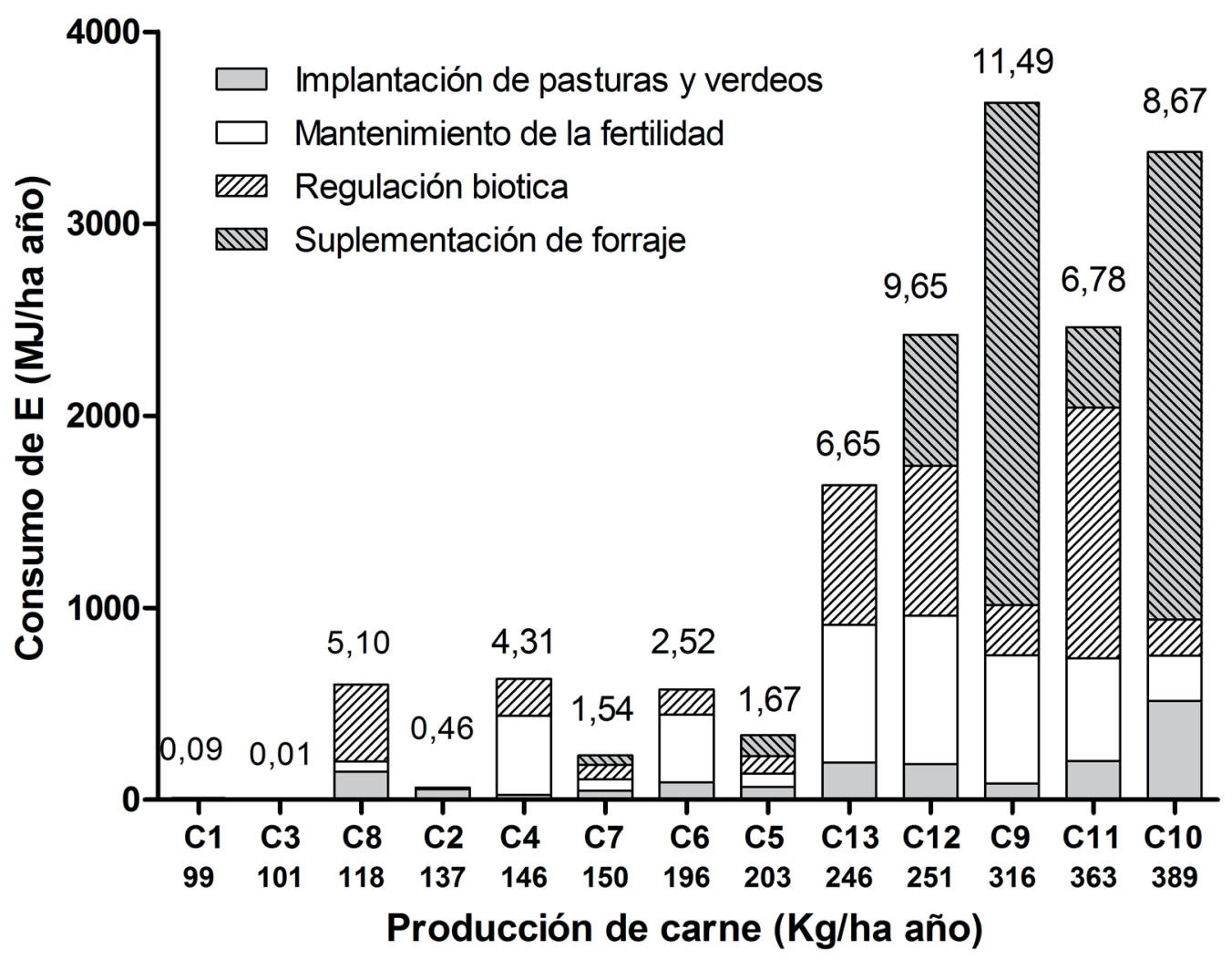

Figura 3. Energía fósil (MJ.ha- ${ }^{-1}$ año-1) consumida según un gradiente de producción secundaria $\left(\mathrm{kg} \cdot \mathrm{ha}^{-1}\right.$.año-1) de 13 establecimientos ganaderos de la cuenca del Salado. En cada caso se consigna la cantidad de energía fósil consumida para producir un kilo de peso vivo de carne $\left(\mathrm{MJ}_{\mathrm{kg}} \mathrm{kg}^{-1}\right.$.

et al. (2006) para la región pampeana. Sin embargo, en la comparación con los valores de referencia propuestos por estos autores se debe tener en cuenta que surgieron de evaluar establecimientos dedicados a diversas actividades agrícolas y ganaderas que incluyó sistemas más intensivos (tambos, agricultura continua, entre otras), por lo que el valor máximo de consumo energético del universo considerado fue muy superior (30.000 $\mathrm{MJ} \mathrm{ha}^{-1}$ año-1). Por esta razón, a pesar de que el consumo energético de los establecimientos más intensivos en el uso de insumos se ubica debajo del menor rango establecido por Viglizzo et al. (2006), para el universo de sistemas de producción de carne pastoril en un área de pastizales naturales de buena productividad potencial, estos valores pueden considerarse elevados.

Dentro del grupo de los establecimientos que consumen menor cantidad de energía fósil (casos 1 a 8), se observa que en siete (casos 1 a 7 ) de los ocho casos el pastizal natural constituyó el principal recurso forrajero, ya que ocupó más del
$73 \%$ de la superficie ganadera, mientras que en el caso restante (caso 8) el pastizal conservado no superó el 43\% (Tabla 2). Se encontró una relación lineal positiva entre la proporción de pastizal y su condición ( $\left.r^{2}=0,74 ; F=31,11 ; p<0,001\right)$, ya que en los establecimientos en que la proporción de pastizal fue mayor al 73\% la condición del pastizal fue más alta, con valores que alcanzaron entre 62 y $75 \%$, mientras que en los establecimientos en que la proporción de pastizal conservado fue inferior al $43 \%$, la condición del pastizal fue más baja. La condición del pastizal, que surge de integrar la cobertura del suelo con la proporción de especies de valor forrajero y de especies nativas, está determinada en gran medida por la metodología de utilización del pastizal, ya que en los casos 1 a 7 se maneja mediante pastoreo controlado. Esta metodología de pastoreo consiste en subdividir la superficie en ambientes homogéneos según el tipo de comunidad vegetal y aplicar eventos de defoliación de intensidad y duración variables y descansos estratégicos según las características de 
las especies clave de cada comunidad (Deregibus et al., 1995). Mediante pastoreo controlado se recuperan las especies de mayor valor forrajero en campos degradados como consecuencia del pastoreo continuo, se incrementa el valor zootécnico del pastizal y por lo tanto aumenta la producción secundaria (Jacobo et al., 2006). Además, en pastizales halomórficos esta metodología de pastoreo incrementa la cobertura vegetal y mejora las propiedades de los suelos, ya que aumenta el contenido de carbono orgánico, reduce la salinidad y el pH y mejora la tasa de infiltración (Vecchio, 2014). Si bien en los establecimientos seleccionados se encontró una fuerte relación entre la proporción de pastizal natural y su condición, éste no es un resultado generalizable ya que el pastoreo controlado no es una práctica habitual en la cuenca del Salado (Rodríguez \& Jacobo, 2012). Por esta razón, un manejo adecuado del pastoreo resulta una práctica indispensable si se desea intensificar la producción con poca utilización de insumos y por lo tanto bajo consumo de energía fósil. La aplicación de tecnologías de procesos tales como el pastoreo controlado permitiría minimizar la cantidad de energía fósil utilizada en el mantenimiento de la fertilidad o en el control de malezas, plagas y enfermedades, debido a que dinamizan procesos tales como el ciclado de nutrientes o la regulación biótica (lermanó, 2015).

Como resultaba esperable, a medida que se incrementó el consumo de energía fósil (entrada), la energía producida (salida) fue mayor debido a la mayor producción secundaria (Figura 4). La ordenada al origen de la regresión entre entradas y salidas de energía de los 13 casos de estudio indica que aún en los casos en que no exista ningún consumo de energía fósil (es decir, no se apliquen insumos ni se realicen ninguna de las prácticas de las consideradas en este trabajo), la producción de carne -y en consecuencia la salida de energíatendrá valores positivos, como sucede cuando la producción de carne se basa sólo en el uso del pastizal natural. La pendiente de esta regresión fue menor a uno, por lo tanto el incremento en la energía producida fue menos que proporcional a la energía invertida. La línea punteada de la figura,

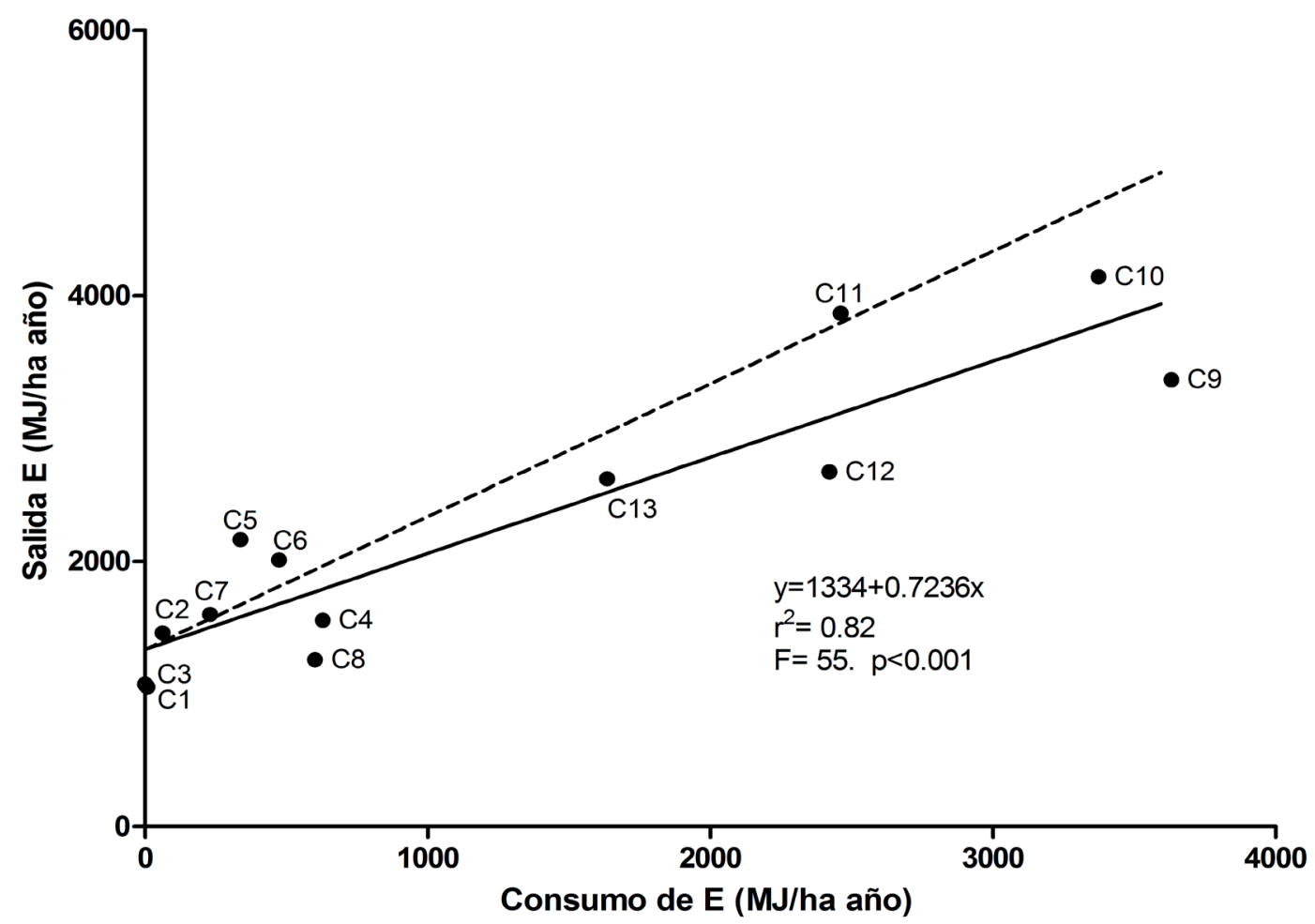

Figura 4. Relación entre la energía fósil consumida (entrada de energía) y la energía producida, estimada mediante la producción de carne (salida de energía) en 13 establecimientos ganaderos de la Cuenca del Salado. La línea entera indica la regresión lineal entre ambas variables. Se inserta el resultado de la regresión. La línea punteada indica la relación 1:1 a partir del valor de la ordenada al origen para la cual a un incremento de la entrada de energía fósil le corresponde un incremento igual de la salida energética. 
que indica la relación 1:1, es la línea teórica en la cual a un incremento de las entradas de energía fósil al sistema le corresponde un incremento igual de la salida energética, calculada de acuerdo al contenido de energía de la carne. Los establecimientos que están sobre el eje de ordenadas y sobre o por encima de la línea que representa la relación 1:1 son aquellos que convierten en carne de manera proporcional, o más que proporcional, la energía fósil invertida, y se caracterizan por producir prácticamente sobre pastizal natural (casos 1, 2 y 3), o sembrar una pequeña parte de su superficie con cultivos anuales ofrecidos en pastoreo directo o como silo (casos 5 y 6), o realizar una invernada pastoril (caso 11), único representante de los campos reunidos en los grupos de alto consumo. La buena conversión de la energía fósil observada en el caso 11 está en relación al sistema de producción, ya que en el proceso de invernada todos los nutrientes se asignan al crecimiento de los animales y por lo tanto a la producción de carne, mientras que en el resto de los casos que incluyen la etapa de cría, alrededor del $60 \%$ de los nutrientes se asignan al mantenimiento del plantel de vacas y toros (Di Marco, 1998).

En los establecimientos reunidos en el grupo de menor consumo energético no se observó un aumento de consumo a medida que la producción de carne aumentó de 99 a $203 \mathrm{~kg} \mathrm{ha}^{-1}$ año-1 (Figura 3); esto deja en evidencia la posibilidad de incrementar la producción de carne en este rango con mínimos consumos energéticos. Además de utilizar el pastizal natural mediante pastoreo controlado, la siembra de una pequeña superficie de los suelos no inundables con cultivos anuales o pasturas perennes y/o la oferta de pequeñas cantidades de forrajes conservados, permitieron alcanzar alta producción de carne con mínimos aportes energéticos, tales como los casos 5 y 6 , donde se obtuvieron alrededor de $200 \mathrm{~kg} \mathrm{ha}^{-1}$ año-1 de carne. En el grupo de menor consumo, el uso de energía fósil por unidad de producto no superó los 5,10 MJ $\mathrm{kg}^{-1}$ de carne, con valores extremadamente bajos como 2,52 y 1,67 MJ kg-1 de carne en los casos 5 y 6 , respectivamente, y con valores menores a $1 \mathrm{MJ} \mathrm{kg}^{-1}$ en los casos 1, 2 y 3. La energía necesaria para producir un kilo de peso vivo bovino de estos últimos casos resultó semejante a los 0,5 MJ por kilo de peso vivo calculados a partir de los resultados de Cieza \& Flores (2007) en sistemas de producción ganadera sobre pastizales de la misma zona. El consumo energético de diversos sistemas ganaderos para obtener un kilo de producto es extremadamente variable. Desde $169 \mathrm{MJ}$ $\mathrm{kg}^{-1}$ de carne en sistemas muy intensificados como el de Japón, donde la cría y el engorde se realizan a corral y los alimentos se transportan desde lejos (Ogino et al, 2007), $121 \mathrm{MJ} \mathrm{kg}^{-1}$ de carne en un sistema pastoril con pasturas de alta producción y gran utilización de heno (Pelletier et al., 2010) a $22 \mathrm{MJ} \mathrm{kg}^{-1}$ de carne en sistemas como los de producción orgánica de Suecia, basados en la siembra de pasturas y verdeos (Cederberg \& Stadig, 2003). Con respecto a estos valores, aun los establecimientos más intensificados de nuestro estudio (casos 9 y 10) fueron más eficientes en el uso de energía fósil, ya que consumieron 8,67 y 11,49 MJ $\mathrm{kg}^{-1}$ respectivamente, probablemente debido al aporte del pastizal natural o de las pasturas naturalizadas de festuca, que no requieren insumos para garantizar su producción.

Al relacionar la eficiencia en el uso de la energía fósil con la condición del pastizal ponderada por su superficie relativa (Figura 5), el grupo que consume menor cantidad de energía fósil (casos 1 a 8) presentó valores de eficiencia muy por encima del límite de 2, que indica que no existe riesgo ambiental, según Viglizzo et al. (2006). Dentro de este grupo se discriminaron siete casos (1 a 7) cuya condición del pastizal ponderada por su superficie relativa fue superior a 50 como resultado de que el pastizal natural representa más del $73 \%$ de la superficie y la condición de estos pastizales es su-

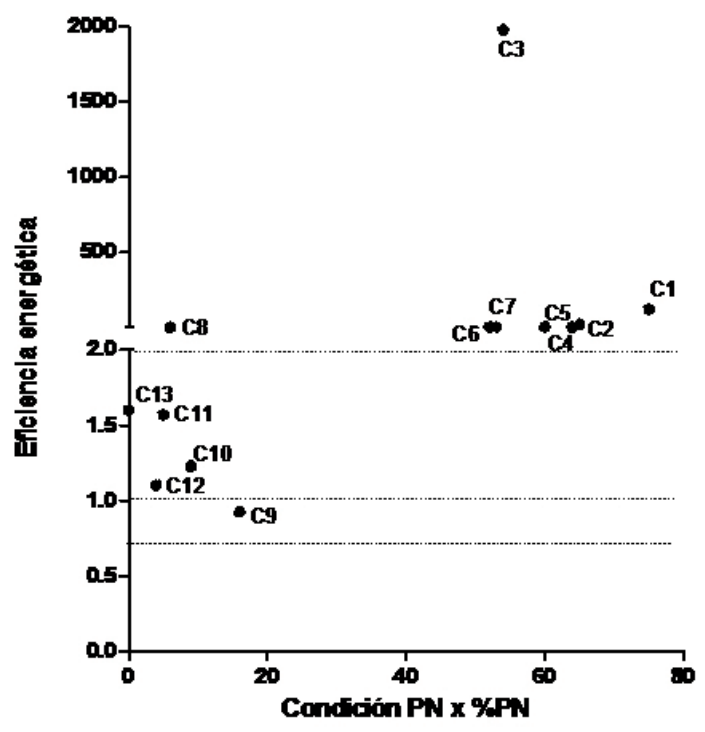

Figura 5. Eficiencia energética (energía contenida en la carne producida en un año/energía fósil consumida en un año) en relación a la participación del pastizal natural en la alimentación animal (proporción de pastizal natural * condición del pastizal natural) de 13 establecimientos ganaderos de la cuenca del Salado. Se indican con líneas horizontales los inversos multiplicativos de los rangos de valores de referencia de Viglizzo et al. (2006): Sin problema >2, Alerta 1-2; Riesgo leve 0,66-1 
perior al $60 \%$, mientras que uno de los casos (caso 8) presentó un bajo valor de condición del pastizal ponderada por su superficie relativa, ya que sólo el $16 \%$ de su superficie corresponde a pastizal natural y su condición fue inferior a 40 (Figura 5, Tabla 2). Los otros dos grupos de mayor consumo energético (casos 9 al 13) presentaron valores de eficiencia en el uso de la energía fósil menor a 2 (cuatro casos se ubicaron en el rango de alerta -1 a 2 - y un caso en el rango riesgo leve -0,66 a 1-, según Viglizzo et al., 2006). El caso 9, cuya eficiencia energética es menor a 1, evidencia que por cada unidad de energía fósil consumida no alcanza a producirse una cantidad equivalente de energía contenida en el producto animal. Estos casos más intensificados cuentan con menos de $43 \%$ de su superficie de pastizal natural cuya condición es menor a 40 (Tabla 2). Estos resultados demuestran una fuerte incidencia de la contribución del pastizal natural y de su estado de conservación sobre la eficiencia en el uso de la energía. Entre los casos con eficiencia en el uso de la energía fósil mayor a 2 hay establecimientos dedicados tanto a la cría (casos 1, 3, 4 y 8) como a la cría y recría (casos 5, 6 y 7 ), y aun al ciclo completo (caso 2), mientras que los casos con valores de eficiencia energética menores a 2 corresponden a establecimientos de ciclo completo (casos 9, 10, 12 y 13) o invernada (caso 11). Los altos valores de eficiencia energética obtenidos en los sistemas ganaderos que se basan en el pastizal natural son consistentes con los datos de Gil et al. (2009), quienes informaron valores de eficiencia energética de 20 en sistemas ganaderos de cría bovina sobre pastizal natural de la provincia de San Luis, y con los hallados por lermanó (2015) en sistemas agrícola-ganaderos de la región pampeana, que estimó valores de eficiencia energética de 9,3.

El proceso de intensificación ganadera en la cuenca del Salado, que consiste en el reemplazo de la vegetación natural por pasturas y verdeos, conduce a la reducción de la superficie de pastizal natural en la región y al empeoramiento de la condición del pastizal remanente. Una alta proporción de los suelos de la región presenta severas limitaciones para la implantación de recursos forrajeros anuales o perennes, por lo que los recursos implantados no pueden expresar su potencial productivo y manifiestan una baja estabilidad (Rodríguez \& Jacobo, 2010). En los casos analizados se observó una relación inversa entre la superficie y la condición del pastizal remanente, ya que cuando el recurso pastizal contribuyó en baja proporción a la alimentación del ganado no se aplicó pastoreo controlado, lo que determina el deterioro de la condición del pastizal y la disminución de su re- ceptividad (Jacobo et al. 2006). Estos dos factores (la baja productividad y estabilidad de los recursos implantados y la baja receptividad del pastizal remanente) explican, al menos parcialmente, que el aumento de la producción secundaria de los establecimientos más intensificados sea menos que proporcional al aumento del consumo de energía fósil, lo que determina la disminución de la eficiencia en el uso de la energía.

La provisión de servicios ecosistémicos de los pastizales naturales depende de su estado de conservación (Briske et al., 2005). La reducción de la superficie y deterioro de la condición del pastizal causada por el proceso de intensificación afecta la provisión de servicios ecosistémicos de regulación y soporte (Altesor, 2010; Power, 2010; Soussana et al., 2010) ya que provoca la disminución de la diversidad florística y de la productividad primaria, altera su estacionalidad (Jacobo et al., 2006; Rodríguez \& Jacobo, 2010; 2013) y reduce su estabilidad y resiliencia (Milchunas \& Lauenroth, 1993). Dado que la estimación de los flujos de energía permite cuantificar la provisión de servicios ecosistémicos (Ferraro, 2011), la alta eficiencia energética de los sistemas basados en pastizales naturales en buena condición debido al método de pastoreo da cuenta de su mayor capacidad de proveer estos servicios con respecto a los sistemas que hacen un uso más intensivo de insumos.

\section{CONCLUSIONES}

Los resultados obtenidos indican que el proceso de intensificación en la cuenca del Salado, consistente en el reemplazo de la vegetación natural por la siembra de pasturas y verdeos, conduce a la disminución de la eficiencia energética de los sistemas de producción de carne. Las alternativas para mantener niveles aceptables de producción de carne con bajos gastos energéticos son: a) conservar una elevada proporción de pastizal en buena condición mediante el manejo del pastoreo, o b) combinar lo anterior con la siembra de cultivos anuales y/o pasturas ofrecidos en pastoreo directo o como reservas en los suelos aptos para la agricultura, que en esta región representan una pequeña proporción de la superficie. Con estas estrategias se aprovecha la elevada potencialidad productiva del pastizal de la región y se minimiza el riesgo sobre la provisión de servicios ecosistémicos. 


\section{AGRADECIMIENTOS}

Los autores agradecen muy especialmente a los productores y asesores que contestaron las encuestas y abrieron las puertas de sus establecimientos y a los consultores anónimos que con sus sugerencias nos permitieron mejorar la calidad del trabajo. Este trabajo se realizó con fondos provenientes de la Secretaría de Ciencia y Técnica de la Universidad de Buenos Aires (Proyecto UBACyT 01/Q339)

\section{BIBLIOGRAFÍA}

Altersor, A. 2010. Servicios ecosistémicos de los pastizales naturales. En Altesor, A., W. Ayala y J.M. Paruelo (Eds,): Bases ecológicas y tecnológicas para el manejo de pastizales. Serie FPTA, INIA, pp 221-234. <http:// www.agro.uba.ar/users/paruelo/libros/FPTA_175.pdf> Consultada el 21/10/2012.

Balvanera, P.; A.B. Pfisterer, N. Buchmann, J.S. He, Nakashizuka, D.Raffaelli and B.Schmid, 2006. Quantifying the evidence for biodiversity effects on ecosystem functioning and services. Ecology letters 9:1146-1156.

Biondini, M.E.; P.W. Mielke Jr and K.J. Berry, 1988. Datadependent permutation techniques for the analysis of ecological data. Vegetatio 75:161-168.

Briske, D.D.; S.D.Fuhlendorf and F.E. Smeins, 2005. State-and-transition models, thresholds, and rangeland health: a synthesis of ecological concepts and perspectives. Rangeland Ecology \& Management 58:1-10.

Cahuepé, M.A.; L.G. Hidalgo y A. Galatoire, 1985. Aplicación de un índice de valoración zootécnica en pastizales de la Depresión del Salado. Revista Argentina de Producción Animal 5:681-690.

Casey, J.W. and N.M. Holden, 2006. Quantification of GHG emissions from sucker-beef production in Ireland. Agricultural Systems 90:79-98.

Cederberg, C. and K. Darelius, 2002. Using LCA methodology to assess the potential environmental impact of intensive meat production. Cederberg, C., Life Cycle Assessment of animal production, Thesis, Department of Applied Environmental Science. Göteborg University, Göteborg, Sweden.

Cederberg, C. and M. Stadig, 2003. System expansion and allocation in life cycle assessment of milk and beef production. The International Journal of Life Cycle Assessment 8:350-356.

Cieza, R. y C.C. Flores, 2007. Sustentabilidad económica y eficiencia energética de las estrategias de diversificación de sistemas productivos de la Cuenca del Salado, Argentina. Rev. Brasileña de Agroecología 2: 264-267.
Deregibus, V.A.; E. Jacobo and A. Rodríguez, 1995. Perspective: Improvement in rangeland condition of the Flooding Pampa of Argentina through controlled grazing. African Journal of Range \& Forage Science 12:92-96.

Di Marco, O. 1998. Crecimiento de vacunos para carne. Di Marco (Ed.), Balcarce, 246 pp.

Ferraro, D.O. 2011. Eficiencia energética y servicios ecosistémicos. En Laterra P., Jobaggy E., Paruelo JM (Eds.) Valoración de servicios ecosistémicos. Conceptos, herramientas y aplicaciones para el ordenamiento territorial. Buenos Aires: Ed. INTA, pp.221-235.

Fisher, B.; R.K. Turner and P. Morling, 2009. Defining and classifying ecosystem services for decision making. Ecological economics 68:643-653.

Frank F, Viglizzo E. Evaluación ecológica: ejemplo de estudio en las pampas de Argentina. Revista de la Cátedra Unesco sobre Desarrollo Sostenible de la UPV/ EHU $N^{\circ} 04 \cdot 2010 .: 79$

Gil, S.B.; M.A. Herrero, M.C. Flores, M.L. Pachoud and M.M. Hellmers, 2009. Intensificación agropecuaria evaluada por indicadores de sustentabilidad ambiental. Archivos de zootecnia 58:413-423.

Halberg, N.; G. Verschuur and G. Goodlass, 2005. Farm level environmental indicators; are they useful?: an overview of green accounting systems for European farms. Agriculture, Ecosystems \& Environment 105:195-212.

lermanó, M.J., 2015. Sistemas mixtos familiares de agricultura y ganadería pastoril de la Región Pampeana: eficiencia en el uso de la energía y rol funcional de la agrobiodiversidad. Tesis doctoral, Facultad de Ciencias Agrarias y Forestales.

Jacobo, E.J.; A.M. Rodríguez, N. Bartoloni and V.A. Deregibus, 2006. Rotational grazing effects on rangeland vegetation at a farm scale. Rangeland Ecology \& Management 59:249-257.

Jacobo, E. y A. Rodríguez, 2009. Valorización de pastizales naturales en ambientes húmedos. Indicadores de sustentabilidad. V Congreso de la Asociación Argentina para el Manejo de los Pastizales Naturales. http:// www.produccion-animal.com.ar/produccion_y_manejo_pasturas/pasturas\%20naturales/163-Valorizacion. pdf > Consultada el 7/3/2016

McCune, B. andM.J. Mefford, 1999. PC-ORD: multivariate analysis of ecological data; Version 4 for Windows;[User's Guide]. MjM Software Design.

Milchunas, D.G. and W. Lauenroth, 1993. Quantitative effects of grazing on vegetation and soils over a global range of environments. Ecological Monographs 6: 327-366.

Ogino, A.; H. Orito, K. Shimada and H. Hirooka, 2007. Evaluating environmental impacts of the Japanese beef cow-calf system by the life cycle assessment 
method. Animal Science Journal 78:424-432.

Paruelo, J.M.; J.P. Guerschman and S.R. Verón, 2005. Expansión agrícola y cambios en el uso del suelo. Ciencia hoy 15:14-23.

Pelletier, N.; R. Pirog and R. Rasmussen, 2010. Comparative life cycle environmental impacts of three beef production strategies in the Upper Midwestern United States. Agricultural Systems 103:380-389.

Perelman, S.B.; E.J. Chaneton, W.B. Batista, S.E. Burkart and R.J. Leon, 2007. Habitat stress, species pool size and biotic resistance influence exotic plant richness in the Flooding Pampa grasslands. Journal of Ecology 95:662-673.

Pérez Neira, D., 2010. Economía, Energía, Retomando el Debate. El Caso aplicado de la Agricultura y Ganadería en Andalucía. Tesis doctoral, Universidad Internacional de Andalucía.

Pimentel, D.; L.E. Hurd, A.C. Bellotti, M.J. Forster, I.N. Oka, O.D. Sholes and R.J. Whitman, 1973. Food production and the energy crisis. Science 182:443-449.

Power, A.G., 2010. Ecosystem services and agriculture: tradeoffs and synergies. Philosophical Transactions of the Royal Society of London B: Biological Sciences, 365: 2959-2971.

Rearte, D., 2010. Situación actual y prospectivas de la producción de carne vacuna. Instituto Nacional de Tecnología Agropecuaria, Buenos Aires (Argentina). Estación Experimental Agropecuaria Balcarce, Buenos Aires (Argentina). Programa Nacional Carnes. Disponible en http://inta.gob.ar/documentos/situacion-actual-y-prospectiva-de-la-produccion-de-carne-vacuna>. Consultada el 26/11/2014.

Recavarren,P.M. y M.J. Martinefsky, 2009. Alerta amarillo: la degradación de los recursos forrajeros. Visión rural. a. 16, No $77: 29-31$.

Rodriguez, A.M. and E.J. Jacobo, 2010. Glyphosate effects on floristic composition and species diversity in the Flooding Pampa grassland (Argentina). Agriculture, Ecosystems \& Environment 138:222-231.

Rodríguez, A.M. y E.J. Jacobo, 2012. Manejo de pastizales naturales para una ganadería sustentable en la pampa deprimida: buenas prácticas para una ganadería sustentable de pastizal: kit de extensión para las pampas y campos. Fundación Vida Silvestre Argentina, Aves Argentinas, 104 pp.

Rodriguez, A.M. and E.J. Jacobo, 2013. Glyphosate effects on seed bank and vegetation composition of temperate grasslands. Applied Vegetation Science 16:51-62.

Savory, A. and J. Butterfield, 1999. Holistic Management: A New Framework for Decision Making. Journal of Range Management 52:543-544.

Soussana, J.F.; T. Tallec and V. Blanfort, 2010. Mitigating the greenhouse gas balance of ruminant production systems through carbon sequestration in grasslands. Animal 4:334-350.

Teague, W.R.; S.L. Dowhower, S.A. Baker, N. Haile, P.B. DeLaune and D.M.Conover, 2011. Grazing management impacts on vegetation, soil biota and soil chemical, physical and hydrological properties in tall grass prairie. Agriculture, Ecosystems \& Environment 141:310-322.

Vázquez, P. y M. Rojas, 2006. Zonificación Agro-ecológica del Área de Influencia de la EEA Cuenca del Salado. Publicación Técnica Nº 2. 17 pp.

Vázquez, P.; M. Rojas and J. Burges, 2008a. Caracterización y tendencias de la ganadería bovina en la cuenca del Salado. Veterinaria Argentina 25:572-584.

Vázquez, P.; S. Masuelli, G. Platzeck y O. Boolsen, 2008b. Recurrencia de anegamiento en la depresión del río Salado: subcuenca B4. Revista de Teledetección de la Asociación Española de Teledetección 30: 47-59

Vecchio, M.C., 2014. Modificaciones en la vegetación y el suelo inducidos por el manejo del pastoreo en la estepa de halófitas de la Pampa Deprimida. Tesis de Magister en Recursos Naturales, Escuela para Graduados Alberto Soriano FAUBA. Argentina.

Viglizzo, 2013. Criterios y parámetros para generar un indicador de conservación de pastizales. https:// www.google.com.ar/webhp?sourceid=chromeinstant\&ion=1\&espv=2\&ie =UTF-8\#q=Criterios $+y+P a$ r\%C3\%A1metros+para+Generar+un+Indicador+de + Conservaci\%C3\%B3n+de+Pastizales>. Consultada el $12 / 08 / 2014$.

Viglizzo, E.F.; F. Frank, J. Bernardos, D.E. Buschiazzo and S. Cabo , 2006. A rapid method for assessing the environmental performance of commercial farms in the Pampas of Argentina. Environmental Monitoring and Assessment 117:109-134.

Wrage, N.; J. Strodthoff,, H.M. Cuchillo, J. Isselstein and M. Kayser, 2011. Phytodiversity of temperate permanent grasslands: ecosystem services for agriculture and livestock management for diversity conservation. Biodiversity and Conservation 20:3317-3339.

Williams, A.; E. Audsley and D. Sandars, 2006. Determining the environmental burdens and resource use in the production of agricultural and horticultural commodities: Defra project report IS0205. Disponible en: https://www.google.com.ar/webhp?sourceid=chromeinstant\&ion $=1 \&$ es $p v=2 \& i e=U T F-8 \# q=+w w w$. silsoe.+cranfield.ac.ukand+www.defra.gov.uk>. Consultada el 15/03/2015.

Zentner, R.P.; G.P. Lafond, D.A. Derksen, C.N. Nagy, D.D. Wall and W.E. May, 2004. Effects of tillage method and crop rotation on non-renewable energy use efficiency for a thin Black Chernozem in the Canadian Prairies. Soil and Tillage Research 77:125-136. 